\title{
A perspective on the extension of stochastic orderings to fuzzy random variables
}

\author{
Inés Couso ${ }^{1}$ Didier Dubois ${ }^{2}$ \\ ${ }^{1}$ Department of Statistics, Universidad de Oviedo, Spain \\ ${ }^{2}$ IRIT - CNRS Université Paul Sabatier - Toulouse, France
}

\begin{abstract}
In this paper we study how to make joint extensions of stochastic orderings and interval orderings so as to extend methods for comparing random variables, from the point of view of their respective location or magnitude, to fuzzy random variables. The main idea is that the way fuzzy random variables are interpreted affects the choice of the comparison methods. We distinguish three views of fuzzy random variables, according to which various comparison methods seem to make sense. This paper offers an approach toward a systematic classification of combinations of stochastic and interval or fuzzy interval comparison methods.
\end{abstract}

Keywords: fuzzy random variables, stochastic ordering, interval ordering, possibility theory

\section{Introduction}

The concept of fuzzy random variable, that extends the classical definition of random variable, was introduced by Féron [24] in 1976. Later on, several authors, and especially Kwakernaak [34], Puri and Ralescu [38], Kruse and Meyer [33], Diamond and Kloeden [21], proposed other variants. More recently Krätschmer [31] surveyed all of these definitions and proposed a unified mathematical formal approach. In all of these papers, a fuzzy random variable is defined as a function that assigns a fuzzy subset to each possible output of a random experiment. The different definitions in the literature disagree on the measurability conditions imposed to this mapping, and in the properties of the output space, but all of them intend to model situations that combine fuzziness and randomness.

A fuzzy random variable taking values on the set of fuzzy numbers whose supports are in the real line then generalizes both the notion of random variable and the notion of interval. An interesting issue is then the following: how to compare the relative magnitude of two fuzzy random variables in agreement with existing methods for comparing the locations of random variables $[16,40]$ and existing methods for comparing intervals [25]?

Like for random sets, there is not a unique intuition behind the various definitions of fuzzy random variables originally proposed in the literature. While Feron [24], Puri and Ralescu [38], Diamond and Kloeden [21] view a fuzzy random variable as the extension of a random set to a random membership function, Kwakernaak [34], and Kruse and Meyer [33] consider that this membership function models the imprecise perception of an ill-known classical random variable. This divergence of views, inherited from the same one existing for random sets directly impacts the choice of suitable definitions for extensions of traditional statistical techniques to fuzzy random variables, as well as for basic notions like independence, and conditioning [12].

In this paper, we consider how the methods for ordering fuzzy random variables from the point of view of their relative locations can be affected by these semantic considerations. By convention in the following, random variables are denoted by $x^{\#}$, $y^{\#}$, sets by capitals $A, B$, etc., random sets by "sharped" capitals $X^{\#}, Y^{\#}$, fuzzy sets by tilded capitals $\tilde{A}, \tilde{B}$, etc., and fuzzy random variables as $\tilde{X}^{\#}, \tilde{Y}^{\#}$. Moreover we denote by $\underline{x}$ the mean value of a random variable $x^{\#}$, and likewise $\underline{X}$ the mean value of a random set $X^{\#}, \underline{\tilde{X}}$ the mean value of a fuzzy random variable $\tilde{X}^{\#}$.

\section{The three understandings of fuzzy random variables}

As pointed out in recent publications $[23,11]$, the notion of set, here a closed interval for simplicity, may be used to model three types of information

- Ontic situation: the precise description of a setvalued entity. For instance, the time interval some action has needed to be performed.

- Epistemic situation: The imprecise description of a deterministic point-valued quantity. For instance a time interval representing an agent 's knowledge about a birth-date.

- Random epistemic situation: The imprecise description of a random point-valued quantity. For instance, a time interval representing the distribution of temperatures in a day.

While the same mathematical entity is needed to represent such situations, in the first case the set represents the collection of its elements (it is conjunctive), and has an ontic flavor (the nature of the represented object is set-theoretic), while in the second case, the set contains mutually exclusive values, one of which is the real one (the set is disjunctive), 
and has an epistemic flavor. The same applies, in the last situation, except that now, we deal with a disjunctive set of (frequentist) probability distributions, one of which being the real one.

The same distinctions can be made for fuzzy sets. A fuzzy interval $\tilde{E}$ can stand for an ontic entity, or an epistemic representation. For instance, in the ontic view, it can be a gradual time interval, where the membership grade $\mu_{\tilde{E}}(r)$ represents to what extent the action performed during $\tilde{E}$ is engaged, with the idea that this engagement gradually starts from the beginning and stops gracefully, rather than abruptly. On the contrary, in the epistemic view, the fuzzy interval is a gradual representation of the agent's uncertainty about an ill-known point value, $\mu_{\tilde{E}}(r)$ being the degree of possibility $\pi_{x}(r)$ that $x=r[22]$. Operationally, $\pi_{x}(r)$ can be viewed as the minimal selling price for a gamble that returns 1 euro if $x=r$, following the view of Walley [43]. Under a random epistemic view, $\tilde{E}$ represents the set of probability distributions $\mathcal{P}(\tilde{E})=\left\{P: \forall A, P(A) \leq \Pi(A)=\sup _{r \in A} \mu_{\tilde{E}}(r)\right\}$, containing the actual distribution.

The history of fuzzy random variables is not simple as it was started by two separate groups with respectively epistemic and ontic views in mind [12]. The first papers are those of Kwakernaak [34, 35] in the late seventies, clearly underlying an epistemic view of fuzzy sets, a line followed up by Kruse and Meyer [33]. They view a fuzzy random variable as a (disjunctive) fuzzy set of classical random variables (those induced by selection functions compatible with the random fuzzy set). It represents what is known about an underlying ill-known random variable. These works can thus be viewed as extending the framework of Dempster's upper and lower probabilities [18] based on a probability space $(\Omega, \mathcal{A}, P)$ and a multivalued mapping $X^{\#}: \Omega \rightarrow \mathcal{I}$, the set of closed intervals of the real line. Here we consider a fuzzy set-valued mapping $\tilde{X}^{\#}$, where $\tilde{X}(\omega)$ defines a possibility distribution restricting the possible values of $x(\omega)$. The degree of possibility that $x^{\#}$ is the random variable underlain by the fuzzzy random variable $\tilde{X}^{\#}$ is

$$
\pi_{\tilde{X} \#}\left(x^{\#}\right)=\inf _{\omega \in \Omega} \mu_{\tilde{X}(\omega)}(x(\omega))
$$

For each level $\alpha \in(0,1]$, the interval $\tilde{X}_{\alpha}(\omega)=\{s \in$ $\left.\mathbb{R}: \mu_{\tilde{X}(\omega)}(s) \geq \alpha\right\}$ is the image of $\omega$ via a multiple valued mapping $\tilde{X}_{\alpha}^{\#}$. It is an epistemic random set. Statistical quantities defined from it are fuzzyvalued. For instance, Kruse and Meyer [33] clearly define the variance of a fuzzy random variable as a fuzzy set of positive reals induced by applying the extension principle to the variance formula. Likewise, the probability of an event becomes restricted by a fuzzy interval in the real line $[6,13]$. The evidence theory counterpart of this view deals with belief functions having fuzzy focal elements [44].

An alternative epistemic view of fuzzy random variables was more recently proposed in the spirit of
Walley [42], in terms of a convex set of probabilites induced on the reals [15]. Now, the fuzzy mapping $\tilde{X}$ is viewed as a conditional possibility distribution $\pi(r \mid \omega)=\mu_{\tilde{X}(\omega)}(r)$, which yields for each $\omega$ a family $\mathcal{P}_{\omega}(\tilde{X})$ of probability measures defined as

$$
\left\{P(\cdot \mid \omega): \forall A, P(A \mid \omega) \leq \Pi(A \mid \omega)=\sup _{r \in A} \mu_{\tilde{X}(\omega)}(r)\right\} .
$$

As a consequence, we have partial information about the probability distribution $P^{\prime}$ induced by $(\Omega, \mathcal{A}, P, \tilde{X})$ on $\mathbb{R}$ for each conditional probability $P(\cdot \mid \omega)$ in the set $\mathcal{P}_{\omega}(\tilde{X})$. In fact, this probability measure is given by the following formula, for a measurable set $B$ :

$$
\begin{gathered}
P^{\prime}(B)=\int_{\Omega} P(B \mid \omega) d P(\omega), \text { where, } \\
P(B \mid \omega) \leq \Pi(B \mid \omega)=\sup _{b \in B} \tilde{X}(\omega)(b), \forall \omega \in \Omega .
\end{gathered}
$$

So we induce a convex set of probability measures on the real line from $(\Omega, \mathcal{A}, P, \tilde{X})$. This credal set turns out to be the one induced by the random set constructed from the fuzzy random variable $\tilde{X}^{\#}$ considering realizations $\tilde{X}_{\alpha}(\omega),(\omega, \alpha) \in \Omega \times[0,1]$, with a probability distribution which is the product $P \otimes \lambda$ between $P$ on $\Omega$ and the uniform one $\lambda$ on the unit interval.

In contrast, the line initiated in the mid-1980's by Puri and Ralescu [38] is in agreement with conjunctive random set theory. A fuzzy random variable is then viewed as a random conjunctive fuzzy set, i.e. a classical random variable ranging in a set of (membership) functions. This line of research has been considerably extended so as to adapt classical statistical methods to functional data $[9,27]$. The main issue is to define a space of functions equipped with a suitable metric structure [21, 41]. In this theory of random fuzzy sets, a scalar distance between fuzzy sets is instrumental when defining variance viewed as a mean squared deviation from the fuzzy mean value [30], in the spirit of Fréchet. A scalar variance can be established on this basis and it reflects the variability of membership functions. It makes sense if for instance, membership functions are models of linguistic terms and some "term variability" must be evaluated given a set of responses provided by a set of people in natural language. The existence of three views of fuzzy random variables, the one initiated by Kwakernaak and the one proposed by Puri and Ralescu, and the one based on conditional possibility distributions is acknowledged, surveyed and discussed in $[12,11]$.

\section{Interval and stochastic rankings}

In order to compare fuzzy random variables, one must be in a position to compare intervals and to compare random numbers, as well as fuzzy intervals. Moreover fuzzy intervals can also be interpreted as nested random intervals. 


\subsection{Comparing intervals}

Let $A=\left[a_{1}, a_{2}\right]$ and $B=\left[b_{1}, b_{2}\right]$ be two intervals. Comparing the intervals $A$ and $B$, we have four relations $>_{i}, i=1,2,3,4$, defined in [4] as follows:

1. $\left[a_{1}, a_{2}\right] \geq_{1}\left[b_{1}, b_{2}\right] \Leftrightarrow a_{1} \geq b_{2}$

2. $\left[a_{1}, a_{2}\right] \geq_{2}\left[b_{1}, b_{2}\right] \Leftrightarrow a_{1} \geq b_{1}$

3. $\left[a_{1}, a_{2}\right] \geq_{3}\left[b_{1}, b_{2}\right] \Leftrightarrow a_{2} \geq b_{2}$

4. $\left[a_{1}, a_{2}\right] \geq_{4}\left[b_{1}, b_{2}\right] \Leftrightarrow a_{2} \geq b_{1}$.

The relation $\geq_{1}$ is the strongest, $\geq_{4}$ is the weakest, $\geq_{2}$ and $\geq_{3}$ are of intermediary strength.

In the case of ontic intervals, these comparisons are akin to the proposals made by Allen [4] to compare time intervals.

In fact, if $\left[a_{1}, a_{2}\right]$ models an ill-known value $x$ and $\left[b_{1}, b_{2}\right]$ and ill-known quantity $y, x \geq_{1} y$ is a robust inequality since it holds whatever the values of $x$ and $y$ are; $x \geq_{2} y$ expresses a pessimistic attitude (if the higher $x$ and $y$, the better); $x \geq_{3} y$ expresses an optimistic attitude; while $x \geq_{4} y$ expresses an adventurous attitude, since it may well be that $y>$ $x$ when their values are known. These relations are known in the literature. Denote $A=\left[a_{1}, a_{2}\right]$ and $B=\left[b_{1}, b_{2}\right]$ for short.

- $A>_{1} B \Leftrightarrow \neg\left(B \geq_{4} A\right)$. The strict relation $>_{1}$ is an interval order (Fishburn [25]). In the case of independence between random variables $a$ and $b, P(a>b)=1$ is generally equivalent to $\operatorname{Support}(a)>_{1} \operatorname{Support}(b)$.

- The simultaneous use of $\geq_{2}$ and $\geq_{3}: A \succeq B$ if and only if $A \geq_{2} B$ and $A \geq_{3} B$. This is the canonical order induced by the lattice structure of intervals, equipped with the operations max and min extended to intervals:

$A \succeq B \Leftrightarrow \max \left(\left[a_{1}, a_{2}\right],\left[b_{1}, b_{2}\right]\right)=\left[a_{1}, a_{2}\right] \Longleftrightarrow$ $\min \left(\left[a_{1}, a_{2}\right],\left[b_{1}, b_{2}\right]\right)=\left[b_{1}, b_{2}\right]$ (we call it lattice interval order).

It makes sense to use the latter ordering when comparing non-independent quantities $x$ and $y$. For instance, if $x$ and $y$ depend on a parameter $\lambda$, so that $x=\lambda a_{1}+(1-\lambda) a_{2}$ and $y=\lambda b_{1}+(1-\lambda) b_{2}$, then $x>y, \forall \lambda$ implies $x \succeq y$, not $x>_{1} y$.

\subsection{Stochastic orderings}

Consider two random variables $X: \Omega \rightarrow \mathbb{R}$ and $Y: \Omega \rightarrow \mathbb{R}$ defined on the same probability space $(\Omega, \mathcal{A}, P)$. There are many kinds of stochastic orderings, most of which are presented in detail by Shaked and Shantikumar [40]. In this paper, we consider those that pertain to the relative location of random quantities, i.e. comparing their respective magnitudes. There are three often found methods to compare random variables according to this standpoint:

1. First order stochastic dominance [28]: $X$ dominates $Y$ if $P(X>x) \geq P(Y>x), \forall x \in \mathbb{R}$, or equivalently, when $P_{1}(x, \infty) \geq P_{2}(x, \infty), \forall x \in$
$\mathbb{R}$, where $P_{1}$ and $P_{2}$ respectively denote the probability measures induced by each variable. We will denote it $X \geq_{1 s t} Y$.

2. Dominance in the sense of expected utility [39]: Given an increasing function $u: \mathbb{R} \rightarrow \mathbb{R}, X$ dominates $Y$ wrt $u$ if $E_{P}(u(X)) \geq E_{P}(u(Y))$. We will denote it $X \geq_{u} Y$. It is well known that $X \geq_{1 s t} Y$ if and only if $X \geq_{u} Y$, for all increasing utility functions $u: \mathbb{R} \rightarrow \mathbb{R}$. A special case is Dominance in Expectation: $X$ dominates $Y$ if $E_{P}(X) \geq E_{P}(Y)$. This relation represents the particular case of the previous one, when the utility function $u$ is the identity function $u(x)=x, \forall x \in \mathbb{R}$.

3. Statistical preference [16]: $X$ is statistically preferred to $Y$ if $P(X>Y)+0.5 P(X=$ $Y) \geq 0.5$. It is clear that the above inequality is equivalent to $P(X>Y) \geq P(Y>X)$. It is also related to the sign of the median of the difference $X-Y$. In fact, in [14] the following sequence of implications is established:

$\operatorname{Me}(X-Y)>0 \Rightarrow P(X>Y)>P(Y>X)$

$\Rightarrow P(X>Y) \geq P(X>Y) \Rightarrow \operatorname{Me}(X-Y) \geq 0$.

Although very common in the decision-theoretic literature for the construction of valued preference relations [17], or voting theory (it is the Condorcet pairwise dominance method), the last comparison method is not considered in [40].

\section{Three different procedures for ranking FRVs}

One may use the three interpretations of fuzzy random variables to justify methods for ranking them. For instance, under the ontic view in the line of Puri and Ralescu, the problem of comparing fuzzy random variables is one of randomizing some existing ordering between fuzzy sets representing objective entities. In that case we assume that the fuzzy set ordering is given by considerations related to the application. In contrast, if the fuzzy random variable represents a fuzzy set of ill-known random variables, it sounds more natural to start with a suitable comparison method for comparing random variables, and extend it to the interval or the fuzzy interval case, for instance via the extension principle. In the third view of the fuzzy random variable as a convex set of probabilities, one can apply comparison techniques coming from imprecise probability theory, in the spirit of what has been done recently in [10]. Alternatively, we can then turn the fuzzy random variable into a random set, using $\alpha$-cuts, and enlarging the probability space.

\subsection{Comparison of ontic fuzzy random variables}

Here we assume that there exists some natural way of comparing fuzzy intervals understood as representing gradual objects. This is a direct extension 
of the theory of random sets as described by Kendall [29] or Matheron [36]. We consider a general notion of ranking of fuzzy objects including:

1. (Partial) pre-orders. For any pair of fuzzy objects, some of the following situations will take place: $\tilde{A}$ is strictly preferred to $\tilde{B}, \tilde{B}$ is strictly preferred to $\tilde{A}, \tilde{A}$ and $\tilde{B}$ are equivalent, or $\tilde{A}$ and $\tilde{B}$ are incomparable. Thus, it can be identified with a 4 -valued mapping defined on the collection of pairs of fuzzy objects.

2. Fuzzy preference relations, i.e., mappings assigning a value $R(\tilde{A}, \tilde{B}) \in[0,1]$ to each pair of fuzzy objects, expressing to what extent $\tilde{A}$ is at the right side of $\tilde{B}$. Such a value indicates the degree of preference of the first object over the second one.

If we wish to extend expectation dominance to this case, we can first compute the fuzzy averages of $\tilde{X}^{\#}$ and $\tilde{Y}^{\#}$, which are fuzzy objects $\underline{\tilde{X}}$ and $\underline{\tilde{Y}}$, and use one of the above methods to compare these fuzzy expected objects.

Using partial preorders, one may for instance first defuzzify the objects in some way and get intervals. It can be done by taking the average cut of the fuzzy interval $\underline{\tilde{X}}$, namely, the Aumann integral [5] $\underline{X}=$ $\int_{0}^{1} \tilde{X}_{\alpha} d \alpha$ with respect to the uniform distribution, where $\underline{\tilde{X}}_{\alpha}$ is the $\alpha$-cut of $\underline{\tilde{X}}$. Then, we can indeed distinguish between the following situations:

- $\tilde{X}^{\#}$ strictly expectation-dominates $\tilde{Y}^{\#}\left(\tilde{X}^{\#}>\right.$ $\left.\tilde{Y}^{\#}\right)$ if $\inf \underline{X}>\sup \underline{Y}$

- $\tilde{X}^{\#}$ and $\tilde{Y}^{\#}$ are expectation-equivalent if $\underline{X}=$ $\underline{Y}$

- $\underline{\tilde{X}}^{\#}$ and $\tilde{Y}^{\#}$ are expectation-incomparable if neither inf $\underline{X} \geq \sup \underline{Y}$ nor inf $\underline{Y} \geq \sup \underline{X}$.

Note that taking the average cut of the Puri-Ralescu fuzzy expectation of $\tilde{X}^{\#}$ yields the same result as taking the random set expectation of the average cut of the fuzzy realizations $\tilde{X}(\omega)$ 's. This kind of comparison of fuzzy random variables is proposed in [37].

A natural way of extending this view with degrees is to consider a usual degree of overlap of $\underline{\tilde{X}}$ and $\underline{\tilde{Y}}$ as

$$
o v(\underline{\tilde{X}}, \underline{\tilde{Y}})=\sup _{r} \min \left(\mu_{\underline{\tilde{X}}}(r), \mu_{\underline{\tilde{Y}}}(r)\right) .
$$

It measures the degree of consistency between $\underline{\tilde{X}}$ and $\underline{\tilde{Y}}$ after Zadeh [45]. Then we can compute the degree of expectation-dominance of $\tilde{X}^{\#}$ over $\tilde{Y}^{\#}$ as

$$
R\left(\tilde{X}^{\#}, \tilde{Y}^{\#}\right)=\left\{\begin{array}{l}
1-\operatorname{ov}(\underline{\tilde{X}}, \underline{\tilde{Y}}) \text { if } \inf \underline{\tilde{X}}_{1}>\sup \underline{\tilde{Y}}_{1} \\
0 \text { otherwise }
\end{array}\right.
$$

Then, $R\left(\tilde{X}^{\#}, \tilde{Y}^{\#}\right)=1$ really means that the average fuzzy set $\underline{\tilde{X}}$ is at the right of $\underline{\tilde{Y}}$.

All methods for comparing fuzzy objects lend themselves easily to an extension of statistical preference to fuzzy random variables $\tilde{X}^{\#}$ and $\tilde{Y}^{\#}$. Instead of computing average fuzzy sets, we apply the above comparison to each pair $(\tilde{X}(\omega), \tilde{Y}(\omega))$, and we compute

$$
S P\left(\tilde{X}^{\#}>\tilde{Y}^{\#}\right)=P(\{\omega: \tilde{X}(\omega)>\tilde{Y}(\omega)\})
$$

This approach is proposed by Montes et al. [37] at the generic level. Special cases of this approach for generalizing statistical preference to fuzzy random variables can be found in [3], where one computes $P(\{\omega: R(\tilde{X}(\omega), \tilde{Y}(\omega))>\alpha\})$. In particular, the degree of overlap ov $(\underline{\tilde{X}}, \underline{\tilde{Y}})$ is replaced by other fuzzy interval comparison indices, including several ones where the two fuzzy sets $(\tilde{X}(\omega), \tilde{Y}(\omega))$ are viewed as nested random sets. In [2], the condition $X(\omega)>\tilde{Y}(\omega)$ is tested based on the interval comparison of average cuts, or the Hurwicz-equivalent of average cuts.

In the case where a degree of dominance between fuzzy intervals is provided, we can also compute the average preference degree:

$$
S P\left(\tilde{X}^{\#}>\tilde{Y}^{\#}\right)=\int_{\Omega} R(\tilde{X}(\omega), \tilde{Y}(\omega)) d P(\omega)
$$

In any case, $\tilde{X}^{\#}$ statistically dominates $\tilde{Y}^{\#}$ if $S P\left(\tilde{X}^{\#}>\tilde{Y}^{\#}\right)>0.5$.

For stochastic dominance, we need to define the meaning of the comparison between a fuzzy ontic interval and a real-valued threshold $c$. It can be done in a way similar to the case of comparing two fuzzy intervals as the comparison we need is a special case thereof.

For instance, we can be interested in checking whether $\tilde{X}(\omega)$ lies in the area beyond $c$, by testing whether inf $\tilde{X}(\omega)>c$ or not. A less demanding criterion is to check whether $\tilde{X}(\omega)$ does not lie in the area before $c$, by testing whether $\sup \tilde{X}(\omega)>c$ or not. If the meaning of $\tilde{X}(\omega)>c$ is fixed, then the stochastic dominance can be defined as usual: $\tilde{X}^{\#}$ stochastically dominates $\tilde{Y}^{\#}$ if $P\left(\tilde{X}^{\#}>c\right) \geq$ $P\left(\tilde{Y}^{\#}>c\right)$. Alternatively, we can consider the extent to which fuzzy intervals $\tilde{X}(\omega)$ is strongly above $c$ as:

$$
R_{*}(\tilde{X}(\omega), c)=1-\mu_{\tilde{X}(\omega)}(c) \text { if inf } \tilde{X}(\omega)_{1}>c ;
$$

Likewise the extent to which a fuzzy interval $\tilde{X}(\omega)$ is weakly above $c$ is:

$$
\begin{gathered}
R^{*}(\tilde{X}(\omega), c)=\quad \mu_{\tilde{X}(\omega)}(c) \text { if } \sup \tilde{X}(\omega)_{1}<c \\
1 \text { otherwise }
\end{gathered}
$$

Defining an extension of stochastic dominance would need two thresholds: $c$ on the real line, and $\alpha \in[0,1]$ for the degree of dominance: $\tilde{X}^{\#}$ stochastically dominates $\tilde{Y}^{\#}$ if $P(\{\omega: R(\tilde{X}(\omega), c) \geq \alpha\}) \geq$ $P(\{\omega: R(\tilde{Y}(\omega), c) \geq \alpha\}), \forall \alpha \in[0,1], \forall c \in \mathbb{R}$, for 
$R=R^{*}$ or $R_{*}$. Of course we can restrict this property by fixing the threshold $\alpha$ or by fixing two different thresholds $\alpha_{X}$ and $\alpha_{Y}$ in each side of the inequality. Moreover instead of using the same comparison index $R$ we can also use different ones (a weak and a strong) in each side of the inequality. This extension of stochastic dominance has been used in $[1,2]$ under various forms.

\subsection{Comparing fuzzy sets of random variables}

In this situation, the fuzzy random variable has an epistemic flavor. It is viewed as a possibility distribution over a set of classical random variables. The uncertainty pervading the knowledge of random variables must then be propagated over to the results of stochastic comparisons.

A natural idea is then to fuzzify random variable comparisons using the extension principle. For instance suppose the stochastic comparison gives a partial pre-order. After applying the Extension Principle, a possibility distribution defined over the 4-elements universe \{greater, less, equivalent, incomparable $\}$ is obtained. A decision based on such a possibility measure will take place afterwards, which is a known problem in fuzzy preference modeling [26].

More precisely, suppose we decide to compare random variables $x^{\#}$ and $y^{\#}$ by their average $\underline{x}, \underline{y}$ and extend this comparison to fuzzy random variables $\tilde{X}^{\#}$ and $\tilde{Y}^{\#}$. We can compute the degree of possibility of dominance as:

$\Pi\left(\tilde{X}^{\#}>_{a v} \tilde{Y}^{\#}\right)=\sup _{x^{\#}, y^{\#}: \underline{x}>\underline{y}} \min \left(\pi_{\tilde{X}^{\#}}\left(x^{\#}\right), \pi_{\tilde{Y}^{\#}}\left(y^{\#}\right)\right)$

It is easy to see that considering the fuzzy expectation $\underline{\tilde{X}}$ with membership function $\pi_{\tilde{X}}(r)=$ $\sup _{r=\underline{x}} \pi_{\tilde{X}^{\#}}\left(x^{\#}\right)$, then it holds $\Pi\left(\tilde{X}^{\#}>_{a v} \frac{\tilde{Y}^{\#}}{}\right)=$ $\Pi(\underline{\tilde{X}}>\underline{\tilde{Y}})$ where

$$
\Pi(\underline{\tilde{X}}>\underline{\tilde{Y}})=\sup _{r>s} \min \left(\pi_{\underline{\tilde{X}}}(r), \pi_{\underline{\tilde{Y}}}(s)\right) .
$$

Likewise, using a similar approach, we can define degrees of possibility of stochastic dominance: $\Pi\left(\tilde{X}^{\#}>_{S D} \tilde{Y}^{\#}\right)=$

$$
\sup _{x^{\#}, y^{\#}: x^{\#}>_{S D} y^{\#}} \min \left(\pi_{\tilde{X} \#}\left(x^{\#}\right), \pi_{\tilde{Y}}\left(y^{\#}\right)\right)
$$

or of statistical preference: $\Pi\left(\tilde{X}^{\#}>_{S P} \tilde{Y}^{\#}\right)=$

$$
\sup _{x^{\#}, y^{\#}: P\left(x^{\#}>y^{\#}\right)>P\left(y^{\#}>x^{\#}\right)} \min \left(\pi_{\tilde{X}^{\#}}\left(x^{\#}\right), \pi_{\tilde{Y}^{\#}}\left(y^{\#}\right)\right) .
$$

These extensions of stochastic orderings, even if in some sense unsurprizing and expected, have not been found in the literature. Yet, this way of proceeding in the epistemic fuzzy random variable environment is the most natural. Alternatively, one might consider swapping the fuzzy and stochastic component, adopting the previously described methods for comparing ontic fuzzy random sets, replacing the comparison of fuzzy objects by methods for comparing possibility distributions (for instance using methods proposed in [20], or more generally any possibilistic ranking method). However, in that case we are computing the objective probability (related to the sample space $\Omega$ ) of subjective dominance (pertaining to imprecision of information) of one imprecise observation over another. This idea of describing the variability of some subjective gradual dominance looks much less natural that computing the uncertainty pertaining to a populationbased stochastic ordering.

\subsection{Fuzzy random variables as conditional possibilities}

Now we suppose that the fuzzy random variable represents imprecise possibilistic knowledge about an objective conditional probability relating the sample space and the range of the random variable, yielding a credal set on the real line [15]. The extension of stochastic orderings we can use are those previously introduced by the authors [10], whereby a standard method for comparing random variables is combined with a standard method for comparing intervals. Namely, let $\mathcal{H}_{\tilde{X}} \#$ be the credal set

$\left\{P^{\prime}: P^{\prime}(B)=\int_{\Omega} P(B \mid \omega) d P(\omega), P(B \mid \omega) \leq \Pi_{\tilde{X}(\omega)}(B), \forall B\right\}$

induced by the fuzzy random variable $\tilde{X}^{\#}$.

Equivalently, it comes down to enlarging the sample space $\Omega$ to $\Omega \times[0,1]$, equipped with the product measure $P \otimes \lambda$, where $\lambda$ is Lebesgue measure, and consider the random set $\mathbb{X}^{\#}:(\omega, \alpha) \mapsto \tilde{X}_{\alpha}(\omega)$, based on $\alpha$-cuts $[6,15]$. Then we are back to the problem of extending stochastic orderings with interval orderings, or alternatively to randomize interval orderings.

- Randomizing interval orderings. Suppose we have chosen a way of comparing intervals, say an ordering relation $>_{I}$. Then we can

- compare the interval expectations $\underline{\mathbb{X}}$ and $\underline{\mathbb{Y}}$ of $\tilde{X}^{\#}$ and $\tilde{Y}^{\#}$ using $>_{I}$, where

$$
\underline{\mathbb{X}}=\int_{\Omega} \int_{0}^{1} \tilde{X}_{\alpha}(\omega) d \alpha d P(\omega)
$$

For instance, we may be interested in checking, in the spirit of Walley's decision rule [42] whether the lower expectation of $\mathbb{X}^{\#}-\mathbb{Y}^{\#}$ is positive or not, which comes down to computing

$$
\underline{\mathbb{X}}-\underline{\mathbb{Y}}=[\inf \underline{\mathbb{X}}-\sup \underline{\mathbb{Y}}, \sup \underline{\mathbb{X}}-\inf \underline{\mathbb{Y}}] .
$$

Then, this lower expectation is positive whenever inf $\underline{\mathbb{X}}>\sup \underline{\mathbb{Y}}$.

- Statistical Preference: Check if

$$
P \otimes \lambda\left(\left\{(\omega, \alpha): \tilde{X}_{\alpha}(\omega)>_{I} \tilde{Y}_{\alpha}(\omega)\right\}\right)>0.5 .
$$


Depending on the choice of the comparison between intervals (there are four possibilities) we then retrieve the extensions of statistical preference to random intervals proposed by Chanas and colleagues $[7,8]$ for ranking fuzzy intervals viewed as nested random sets.

- Stochastic Dominance: Check if

$$
\begin{aligned}
& P \otimes \lambda\left(\left\{(\omega, \alpha): \tilde{X}_{\alpha}(\omega)>_{I}\{c\}\right\}\right)> \\
& P \otimes \lambda\left(\left\{(\omega, \alpha): \tilde{Y}_{\alpha}(\omega)>_{I}\{c\}\right\}\right),
\end{aligned}
$$

$\forall c \in \mathbb{R}$. Depending on the choice of the comparison between an interval and a precise value (pessimistic, optimistic) we then retrieve the extensions of stochastic dominance to belief functions proposed by Denoeux [19].

- Extending stochastic orderings using intervals. The idea is again to consider specific selections $x_{s}^{\#}$ and $y_{s}^{\#}$ of the random set $\tilde{X}_{\alpha}(\omega)$ and $\tilde{Y}_{\alpha}(\omega)$ and compare them using stochastic orderings. This selection consists in suitable bounds of $\tilde{X}_{\alpha}(\omega)$ and $\tilde{Y}_{\alpha}(\omega)$ : for instance

$-x_{s}^{\#}(\omega, \alpha)=\inf \tilde{X}_{\alpha}(\omega)$, and $y_{s}^{\#}(\omega, \alpha)=$ $\sup \tilde{Y}_{\alpha}(\omega)$ and compare them, which is a randomized form of interval ordering.

$-x_{\lambda}^{\#}(\omega, \alpha)=\lambda \inf \tilde{X}_{\alpha}(\omega)+(1-$ $\lambda) \sup \tilde{X}_{\alpha}(\omega)$, and $y_{\lambda}^{\#}(\omega, \alpha)$, defined likewise, which is a randomized form of Hurwicz ordering.

\section{Conclusion}

This paper has proposed a systematic investigation of the various ways to jointly extend stochastic orderings and interval orderings to fuzzy random variables. We hope to have convinced the reader that several points of view can be envisaged and that the correct approach will depend about the role played by the fuzzy random variable in the given application, depending upon whether it describes a random fuzzy object, or uncertainty about a random variable, or yet as random interval where uncertainty pertains to an ill-perceived conditional probability measure.

Acknowledgement This paper was partially supported by the Labex CIMI at Paul Sabatier University, the first author benefiting from an Excellence Advanced Researcher position. The first author also acknowledges support from the Spanish projects TIN2011-24302 and TIN2014-56967-R.

\section{References}

[1] F. Aiche, D. Dubois, An Extension of Stochastic Dominance to Fuzzy Random Variables, IPMU 2010, LNAI 6178, pp. 159-168 (2010).
[2] F. Aiche, D. Dubois, Possibility and Gradual Number Approaches to Ranking Methods for Random Fuzzy Intervals, IPMU 2012, Part III, CCIS 299, pp. 9-18 (2012).

[3] F. Aiche, M. Abbas, D. Dubois, Chanceconstrained programming with fuzzy stochastic coefficients. FO \& DM 12 (2013), 125-152.

[4] J. F. Allen: Maintaining Knowledge about Temporal Intervals. Commun. ACM 26 (1983), 832843.

[5] J. Aumann, Integral of set valued functions. Journal of Mathematical Analysis and Applications 12 (1965) 1-12.

[6] C. Baudrit, I. Couso, D. Dubois, Joint propagation of probability and possibility in risk analysis: Towards a formal framework, International Journal of Approximate Reasoning 45 (2007) 82105.

[7] S. Chanas, M. Delgado, J. L. Verdegay, M.A. Vila. Ranking fuzzy real intervals in the setting of random sets. Information Sciences, 69 (1993) 201-217.

[8] S. Chanas, P. Zielinski, Ranking fuzzy intervals in the setting of random sets-further results. Information Sciences, 117 (1999) 191-200.

[9] A. Colubi, Statistical inference about the means of fuzzy random variables: Applications to the analysis of fuzzy- and real-valued data. Fuzzy Sets and Systems 160 (2009), 344-356.

[10] I. Couso, D. Dubois, An imprecise probability approach to joint extensions of stochastic and interval orderings International Conference on Information Processing and Management of Uncertainty in Knowledge-based Systems (IPMU 2012), Catania (Italy), Salvatore Greco (Ed.), Springer, 2012.

[11] I. Couso, D. Dubois, Statistical reasoning with set-valued information: Ontic vs. epistemic views. International Journal of Approximate Reasoning Vol. 55 N. 7, p. 1502-1518, 2014.

[12] I. Couso, D. Dubois, L. Sánchez. Random Sets and Random Fuzzy Sets as Ill-Perceived Random Variables, Springer, SpringerBriefs in Computational Intelligence, 2014.

[13] I. Couso, L. Sánchez. Higher order models for fuzzy random variables. Fuzzy Sets and Systems 159 (2008) 237-258.

[14] I. Couso, S. Moral, L. Sánchez, The behavioral meaning of the median. Inf. Sci. 294 (2015), 127138.

[15] I. Couso, L. Sánchez, Upper and lower probabilities induced by a fuzzy random variable, Fuzzy Sets and Systems 165 (2011), 1-23.

[16] H. David, The method of paired comparisons, Griffin's Statistical Monographs \& Courses, vol. 12, Charles Griffin \& D. Ltd., London, 1963.

[17] B. De Baets, H. De Meyer. On the cycletransitive comparison of artificially coupled random variables. Int. J. Approximate Reasoning 47 (2008) 306-322. 
[18] A.P. Dempster. Upper and lower probabilities induced by a multi-valued mapping, The Annals of Mathematical Statistics 38 (1967) 325-339.

[19] T. Denoeux, Extending stochastic ordering to belief functions on the real line, Information Sciences 179 (2009) 1362-1376.

[20] S. Destercke, I. Couso, Ranking of fuzzy intervals seen through the imprecise probabilistic lens, Fuzzy Sets and Systems, in press (doi:10.1016/j.fss.2014.12.009).

[21] P. Diamond and P. Kloeden. Metric Spaces of Fuzzy Sets, World Scientic (Singapore, 1994).

[22] D. Dubois, H. Prade. Possibility Theory, Plenum Press, New York (1988).

[23] D. Dubois, H. Prade, Gradualness, uncertainty and bipolarity: Making sense of fuzzy sets, Fuzzy Sets and Systems 192 (2012) 3-24.

[24] R. Féron. Ensembles aléatoires flous, C.R. Acad. Sci. Paris Ser. A 282 (1976), 903-906.

[25] P. Fishburn, Interval orderings. New York, Wiley, 1987.

[26] J. C. Fodor, M. R. Roubens, Fuzzy preference modelling and multicriteria decision support, Springer Theory and Decision Library, 14, 1994.

[27] G. González-Rodríguez, A. Colubi , M.A. Gil, Fuzzy data treated as functional data. A one-way ANOVA test approach. Computational Statistics and Data Analysis. Vol. 56(4), pp. 943-955, 2012.

[28] J. Hadar, W. Russell, Rules for Ordering Uncertain Prospects, American Economic Review 59, March 1969, 25-34.

[29] D.G. Kendall. Foundations of a theory of random sets, in: E.F. Harding, D.G. Kendall, Stochastic geometry, Wiley, New York, 322-376 (1974).

[30] R. Körner. On the variance of fuzzy random variables, Fuzzy Sets and Systems 92, 83-93 (1997).

[31] V. Krätschmer. A unified approach to fuzzy random variables, Fuzzy Sets and Systems 123 (2001), 1-9.

[32] R. Kruse. On the Variance of Random Sets, Journal of Mathematical Analysis and Applications 122 (1987), 469-473.

[33] R. Kruse and K. D. Meyer. Statistics with vague data, D. Reidel Publishing Company, Dordrecht (1987).

[34] H. Kwakernaak. Fuzzy random variables: Definition and theorems, Information Sciences 15 (1978), 1-29.

[35] H. Kwakernaak, Fuzzy random variables: Algorithms and examples in the discrete case, Information Sciences 17 (1979), 253-278.

[36] G. Matheron, Random sets and integral geometry. Wiley, New York, 1975.

[37] I. Montes, E. Miranda, S. Montes, Stochastic orders for fuzzy random variables, SMPS 2014.

[38] M. Puri and D. Ralescu. Fuzzy random vari- ables, Journal of Mathematical Analysis and Applications 114 (1986), 409-422.

[39] L.J. Savage. The Foundations of Statistics. Wiley (1954); 2nd edition, Dover Publications Inc., New York, 1972.

[40] M. Shaked, J. Shantikumar, Stochastic Orderings, Springer Series in Statistics, 2007.

[41] W. Trutschnig, G. González-Rodríguez, A. Colubi, M.A. Gil. A new family of metrics for compact, convex (fuzzy) sets based on a generalized concept of mid and spread. Inform Sci, 179 (2009) 3964-3972.

[42] P. Walley, Statistical Reasoning with Imprecise Probabilities, Chapman and Hall, London (1991).

[43] P. Walley, Measures of Uncertainty in Expert Systems. Artif. Intell. 83 (1996) 1-58.

[44] J. Yen. Generalizing the Dempster-Shafer theory to fuzzy sets, IEEE Trans. on Systems, Man and Cybernetics, 20 (1990) 559-570.

[45] L. A. Zadeh. Fuzzy sets as a basis for a theory of possibility. Fuzzy Sets and Systems, 1, 3-28, 1978. 